\title{
Antimicrobial spectrum of the antitumor agent, cisplatin
}

\author{
Kellie Joyce, Shruti Saxena, Amanda Williams, Christopher Damurjian, Neil Auricchio, Sabrina Aluotto, \\ Heather Tynan and Arnold L Demain
}

The Journal of Antibiotics (2010) 63, 530-532; doi:10.1038/ja.2010.64; published online 23 June 2010

Keywords: antimicrobial; antitumor agent; cancer; cisplatin

Cisplatin (cisplatinum, cis-diamminedichloroplatinum [II]), is one of the most important anticancer agents used in medicine. Its structure is shown in Figure 1. Owing to its ability to bind to DNA, cause the cross-linking of adjacent intrastrand purines, and interfere with DNA repair, cisplatin is an effective DNA-damaging and anticancer agent. Although cisplatin is nearly curative for testicular cancer and active against ovarian, head and neck cancer, the potential of this drug as a cure for many other types of cancer is limited because of cellular resistance to cisplatin ${ }^{1}$ and cisplatin's toxicity to humans; for example, renal toxicity, emesis, neurotoxicity, bone marrow suppression, anemia and hearing loss. Owing to the toxicity, cisplatin is administered intravenously in low dosage. The inhibition of Escherichia coli by cisplatin was discovered by Rosenberg et al. ${ }^{2-4}$ before it was known to be an effective antitumor agent. They made this discovery while performing an experiment to analyze the effect of an electric field on the growth of bacteria, the experiment involving the use of platinum electrodes.

Although Rosenberg et al. ${ }^{3}$ found that E. coli and other Gramnegative bacteria such as Aerobacter aerogenes, Alcaligenes faecalis, Proteus mirabilis, Pseudomonas aeruginosa, Klebsiella pneumoniae and Serratia marcescens were sensitive to cisplatin, it was unclear whether other bacteria were also inhibited. At a concentration 15-fold higher than that which inhibited cell elongation in E. coli, Gram-positive bacteria such as Streptococcus lactis, Streptococcus faecalis, Staphylococcus aureus, Sarcina lutea and Neisseria catarrhalis were not inhibited. Although other Gram-positive organisms were inhibited by this high concentration of cisplatin, they were much more resistant than E. coli. We felt it important to revisit this situation and determine the antimicrobial spectrum of cisplatin. Since those early days, three yeasts have been reported to be inhibited by cisplatin; that is, Saccharomyces cerevisiae, ${ }^{5}$ Schizosaccharomyces pombe ${ }^{6}$ and Candida albicans. ${ }^{7}$ One mold, Dictyostelium discoideum, has been reported to be sensitive to cisplatin. ${ }^{8}$ However, there has been very little screening effort focusing on the molds. Hence, we felt it important to expand our screening effort to include more filamentous fungi.
Inhibition of Gram-negative bacteria E. coli, A. aerogenes, A. faecalis, P. mirabilis, $P$. aeruginosa, $K$. pneumoniae and $S$. marcescens by cisplatin has been known for almost 45 years. $^{2}$ In our initial tests, we confirmed the sensitivity of E. coli and $S$. marcescens to cisplatin. Table 1 shows such a test with E. coli strains $153_{\mathrm{z}}^{\gamma}$, ZK 650 and C600 $\mathrm{R} 1$ and with $S$. marcescens. Our further experiments showed inhibition of E. coli strains ZY and ESS as well as P. aeruginosa. The effect of

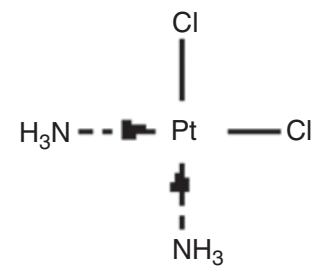

Figure 1 Molecular structure of cisplatin.

Table 1 Inhibition of bacteria by cisplatin ${ }^{a}$

\begin{tabular}{lccc}
\hline Organism & $\begin{array}{c}\text { Agar } \\
\text { medium }\end{array}$ & Day & $\begin{array}{c}\text { Zone size } \\
\text { (mm) }\end{array}$ \\
\hline $\begin{array}{l}\text { Gram-positive } \\
\text { Bacillus brevis 9999 }\end{array}$ & LB & 1 & 13 clear \\
Bacillus cereus 9139 & LB & 1 & 15 clear \\
Bacillus subtilis K & LB & 1 & 15 clear \\
Streptomyces lividans B18 & YEA & 1 & Trace (7 hazy) \\
Gram-negative & & & \\
Escherichia coli C600R1 & & & Trace (7 hazy) \\
Escherichia coli C600R46 & LB & 1 & 11 clear \\
Escherichia coli J53 & LB & 1 & 15 clear \\
Serratia marcescens & LB & 1 & 15 clear
\end{tabular}

Abbreviation: YEA, yeast extract agar.

${ }^{a} 16 \mu \mathrm{g}$ per disc. 


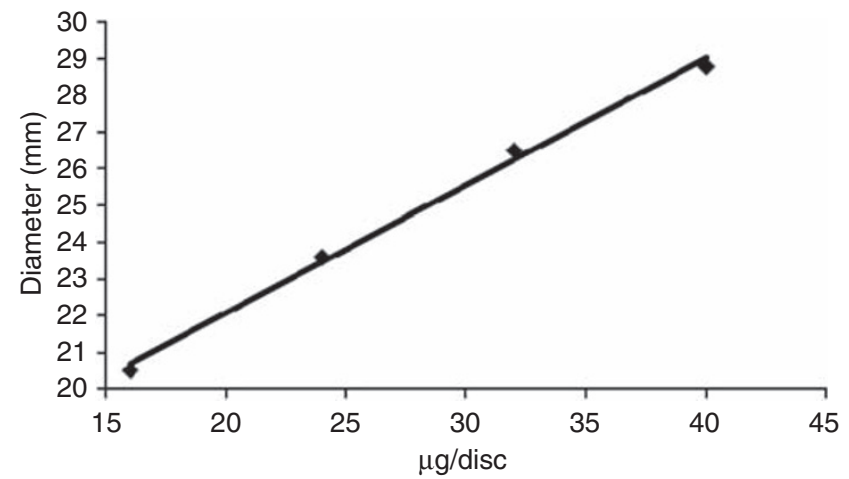

Figure 2 Inhibition of Escherichia coli C600 R1 by increasing doses of cisplatin. Inhibition was measured by the diameter of the clear zones in $\mathrm{mm}$.

Table 2 Cisplatin inhibition of Escherichia coli ZK650 in liquid culture in TSB medium

\begin{tabular}{lccc}
\hline Experiment & Contents of tube & Time & $\begin{array}{c}\text { Growth } \\
\text { (Klett units) }\end{array}$ \\
\hline 1 & No addition & $4.5 \mathrm{~h}$ & 141 \\
& $50 \mu \mathrm{l} \mathrm{DMF}$ & $4.5 \mathrm{~h}$ & 130 \\
2 & $50 \mu \mathrm{l}$ cisplatin in DMFa & $4.5 \mathrm{~h}$ & 25 \\
& No addition & $3.5 \mathrm{~h}$ & 73 \\
& $50 \mu$ l DMF & $3.5 \mathrm{~h}$ & 71 \\
& $50 \mu$ cisplatin in DMFa & $3.5 \mathrm{~h}$ & 19 \\
\hline
\end{tabular}

Abbreviations: DMF, dimethylformamide; TSB, Tryptic Soy broth.

aCisplatin concentration: $60 \mu \mathrm{g} \mathrm{ml}^{-1}$

increasing dosages of cisplatin from 16 to $40 \mathrm{ug}$ per disc against $E$. coli C600 R1 is shown in Figure 2. It can be observed that the increase in zone size is proportional to the cisplatin dosage. We also studied the effect of cisplatin on growth of E. coli ZK650 in liquid medium and found inhibition (Table 2).

Owing to the question in the literature regarding the sensitivity of Gram-positive bacteria to cisplatin, a number of such organisms were tested. Initial testing with Bacillus brevis 9999, Bacillus cereus 9139, Bacillus subtilis $\mathrm{K}$ and the actinomycete Streptomyces lividans B18 revealed inhibition by cisplatin at the lowest dose tested; that is, $16 \mathrm{ug}$ per disc (Table 1). The results against B. subtilis $\mathrm{K}$ using different concentrations of cisplatin varying from 16 to $48 \mathrm{ug}$ per disc, are shown in Figure 3. Later experiments showed inhibition of B. subtilis 168, B. subtilis JH642 and Staphylococcus aureus. The effect of cisplatin on growth of B. subtilis JH642 was also examined. Table 3 shows that cisplatin made up in dimethylformamide inhibited growth at levels of dimethylformamide that were not inhibitory (because of the disk evaporation technique used).

It is known that the yeasts $S$. cerevisiae, ${ }^{5}$ S. pombe ${ }^{6}$ and C. albicans ${ }^{7}$ are inhibited by cisplatin. Our initial studies, shown in Table 4, confirmed cisplatin inhibition of $S$. cerevisiae and $S$. pombe. Five strains of S. pombe; that is, WT, 972, NW158, NW214, NW240 and sp6 were tested and all were found to be inhibited. A later experiment showed inhibition of an additional strain of $S$. pombe; that is, $\operatorname{Rad} 3 \Delta$.

The molds were difficult to test because their growth in liquid culture was filamentous rather than as an evenly distributed turbid suspension. They often grew as pellets, sometimes very large, with or without the apparent presence of sporulation. To solve this

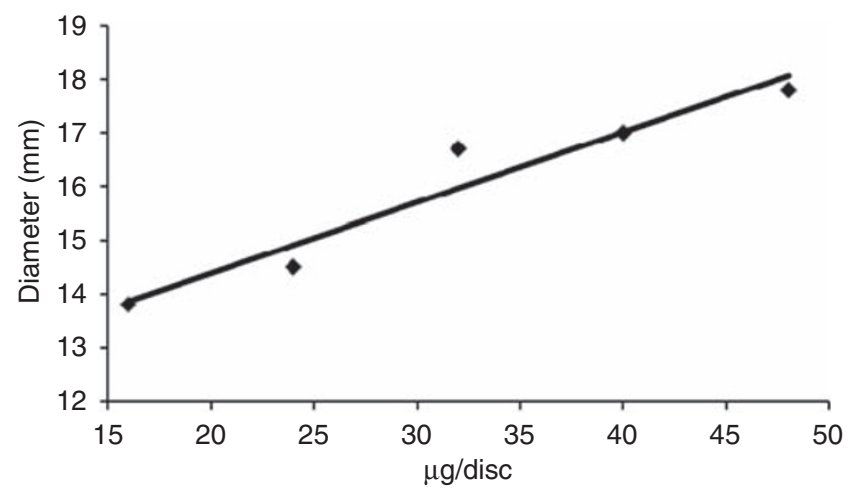

Figure 3 Inhibition of Bacillus subtilis $\mathrm{K}$ by increasing doses of cisplatin. Inhibition was measured by the diameter of the clear zones in $\mathrm{mm}$.

Table 3 Cisplatin inhibition of Bacillus subtilis JH642 in liquid culture in TSB medium

\begin{tabular}{lccc}
\hline Experiment & Contents of tube & Time & $\begin{array}{c}\text { Growth } \\
\text { (Klett units) }\end{array}$ \\
\hline 3 & No addition & $18.5 \mathrm{~h}$ & 149 \\
$225 \mu$ l DMF & $18.5 \mathrm{~h}$ & 155 \\
& $225 \mu$ l cisplatin in DMF* & $18.5 \mathrm{~h}$ & 74
\end{tabular}

Abbreviations: DMF, dimethylformamide; TSB, Tryptic Soy broth.

aCisplatin concentration: $60 \mu \mathrm{g} \mathrm{ml}^{-1}$.

Table 4 Inhibition of fungi by cisplatin ${ }^{\mathrm{a}}$

\begin{tabular}{llcc}
\hline Organism & $\begin{array}{c}\text { Agar } \\
\text { medium }\end{array}$ & Day & $\begin{array}{c}\text { Zone size } \\
\text { (mm) }\end{array}$ \\
\hline Yeasts & & & \\
Saccharomyces cerevisiae YRH499 & SM & 1 & 18 clear \\
Schizosaccharomyces pombe 972 & SM & 2 & Trace (7 hazy) \\
Schizosaccharomyces pombe NW158 & YEA & 3 & 22 clear \\
Schizosaccharomyces pombe NW214 & YEA & 3 & 24 clear \\
Schizosaccharomyces pombe NW240 & YEA & 4 & 41 clear \\
Schizosaccharomyces pombe sp6 & YEA & 4 & 22 clear \\
Molds & & & \\
Alternaria altenata & & & 16 clear \\
Aspergillus niger & SM & 1 & 10 clear \\
Penicillium sp & SM & 1 & Trace (7 hazy)
\end{tabular}

Abbreviations: SM, Sabouraud maltose; YEA, yeast extract agar.

a 16 ug per disc.

problem, small glass beads ( 65 beads per flask) plus either $1.5 \%$ of carboxymethylcellulose or $0.3 \%$ carboxypolymethylene ${ }^{9}$ were added to the flasks. This resulted in a much more homogeneous type of growth. Our initial results showing cisplatin inhibition of Penicillium sp., Alternaria alternata and Aspergillus niger are shown in Table 4. Later experiments revealed cisplatin inhibition of additional molds such as Aspergillus fumigatus, Fusarium oxysporum, Pythium ultimum and Geotrichum candidum.

To summarize, the antitumor agent cisplatin has an extensive antimicrobial spectrum of activity. Growth of all 29 microbes, including seven Gram-negative bacterial strains, eight Gram-positive bacter- 
ial strains, seven yeast strains and seven mold strains was found to be inhibited. This agrees with the point made by Newman and Shapiro ${ }^{10}$ that most antitumor agents have antimicrobial activity. We are now using these cisplatin-inhibitable strains as prescreens to test the antimicrobial activity of novel cisplatin analogs chemically synthesized by Dr Baldwin King and his Drew University students. Those that are antibiotically active will be further tested for antitumor activity in the hope of identifying cisplatin analogs that do not have the toxicity problems associated with cisplatin.

\section{EXPERIMENTAL PROCEDURE}

Organisms were preserved by storage in $30 \%$ glycerol solution at $-80^{\circ} \mathrm{C}$. The cultures were transferred to liquid medium (Tryptic Soy broth for the bacteria and Sabouraud maltose broth for the fungi) at $40 \mathrm{ml}$ per $250 \mathrm{ml}$ flask and grown on the rotary shaker at $28^{\circ} \mathrm{C}$ and 220 r.p.m. until heavy growth was observed. This usually required 2 to 3 days for the bacteria and 4 to 6 days for the fungi. The liquid cultures were then used to seed melted agar media at $500 \mu \mathrm{l}$ per $100 \mathrm{ml}$ of agar medium. The agar media used were as follows: LB agar and Tryptic Soy agar for unicellular bacteria; yeast extract agar for the actinomycete; Sabaroud's maltose agar, Tryptic Soy agar and yeast extract agar for yeasts; and Sabaroud maltose agar as well as Tryptic Soy agar for the molds. The inoculated agar was poured into Petri dishes at $10 \mathrm{ml}$ per plate and allowed to solidify. Cisplatin was dissolved in water up to $5 \mathrm{~mm}$ concentration or in dimethylformamide at higher concentrations (10 and $20 \mathrm{~mm}$ ). Cisplatin was then added to paper discs of $6 \mathrm{~mm}$ diameter, which were allowed to dry on an aluminum foil surface. Those discs that received the high concentration of cisplatin were incubated in a vacuum oven at $42{ }^{\circ} \mathrm{C}$ for $2-3 \mathrm{~h}$ to remove the dimethylformamide before placing them on the inoculated agar surface. Those that received the low concentration of cisplatin could be placed on the agar directly. Plates were incubated at $35-37^{\circ} \mathrm{C}$ for bacteria and $26-28^{\circ} \mathrm{C}$ for fungi until growth was observed. Activity was shown by inhibition zones, the diameters of which were measured.

\section{CONFLICT OF INTEREST}

The authors declare no conflict of interest.

\section{ACKNOWLEDGEMENTS}

We thank Dr Baldwin King for samples of cisplatin and Prakash Masurekar, Douglas Eveleigh and Charles Martin for S. cerevisiae strains. We acknowledge Drew undergraduate student Christian Maggio and high school students Anisha Mukherjee and Allison Dyevoich for laboratory assistance. The work was supported by a grant from AAAS/Merck and financial assistance from the Drew Summer Science Institute (DSSI) and RISE. We acknowledge the encouragement given by former RISE Director, Dr Ashley H Carter, the present Director, Dr Jon Kettenring and colleague Dr Vincent Gullo. Administrative assistance was provided by Miriam Donohue. All the authors of the paper except AL Demain were or are undergraduate students of Drew University.

1 Burger, H. et al. A genome-wide screening in Saccharomyces cerevisiae for genes that confer resistance to the anticancer agent cisplatin. Biochem. Biophys. Res. Commun. 269, 767-774 (2000).

2 Rosenberg, B., Van Camp, L. \& Krigas, T. Inhibition of cell division in Escherichia coli by electrolysis products of a platinum electrode. Nature 205, 698-699 (1965).

3 Rosenberg, B., Renshaw, E., Van Camp, L., Hartwick, J. \& Drobnik, J. Platinum-induced filamentous growth in Escherichia coli. J. Bacteriol. 93, 716-721 (1967).

4 Rosenberg, B., Van Camp, L., Grimley, E. B. \& Thomson, A. J. The inhibition of growth or cell division in Escherichia coli by different ionic species of platinum(IV) complexes. J. Biol. Chem. 242, 1347-1352 (1967).

5 Cardenas, M. et al. Antifungal activities of antineoplastic agents: Saccharomyces cerevisiae as a model system to study drug action. Clin. Microbiol. Rev. 12, 583-611 (1999).

6 Perego, P. et al. Sensitivity to cisplatin and platinum-containing compounds in Schizosaccharomyce pombe Rad mutants. Molec. Pharmacol. 4, 213-219 (1998).

7 Moussa, N., Ghannoum, M. A., Whittaker, P. A., el-Ezaby, M. S. \& Quraman, S. Effects of cisplatin and two novel palladium complexes on Candida albicans. Microbios 62, 165-178 (1990)

$8 \mathrm{Yu}$, S. L., Lee, S. K., Alexander, H. \& Alexander, S. Rapid changes of nucleotide excision repair gene expression following UV-irradiation and cisplatin treatment of Dictyostelium discoideum. Nucleic Acid Res. 26, 3397-3403 (1998).

9 Yang, W., Hartweig, E. A., Fang, A. \& Demain, A. L. Effects of carboxymethylcellulose and carboxypolymethylene on morphology of Aspergillus fumigatus NRRL 2346 and fumagillin production. Curr. Microbiol. 46, 24-27 (2003).

10 Newman, D. J. \& Shapiro, S. Microbial prescreens for anticancer activity. SIM News $\mathbf{5 8}$ 132-150 (2008). 